\title{
ITERATING BONFERRONI BOUNDS
}

\section{Fred M. HOPPE}

Department of Statistics, The University of Michigan, Ann Arbor. Michigan 48109-1027, USA

Received January 1985

\begin{abstract}
A general method is presented for generating improved Bonferroni bounds whenever an improvement on Booles inequality holds.
\end{abstract}

AMS 1980 Subject Classification: 62E99.

Keywords: Bonferroni inequalities.

\section{Introduction}

If $A=\cup{ }_{1}^{n} A_{i}$ where $\left\{A_{i}\right\}$ are arbitrary events then by the principle of inclusion-exclusion

$$
P(A)=S_{1}-S_{2}+S_{3}-\cdots+(-1)^{n-1} S_{n}
$$

where $S_{k}=\sum P\left(A_{i_{1}} A_{i_{2}} \cdots A_{i_{k}}\right)$ and the sum is taken over all distinct subscripts $1 \leqslant i_{1}<i_{2}<\ldots<i_{k} \leqslant n$. For each $m$ the sum of the first $m$ odd (even) terms provide upper (lower) Bonferroni bounds

$$
P(A) \leqslant S_{1}, \quad P(A) \geqslant S_{1}-S_{2}, \quad P(A) \leqslant S_{1}-S_{2}+S_{3}, \quad \ldots
$$

The usual proof (as presented in Feller (1968)) uses a combinatorial identity and gives little insight into the essential simplicity of these inequalities.

We present an elementary proof which has two appealing properties. First, each bound is obtained from its predecessor and ultimately, therefore, it is the first (also called Boole's) inequality

$$
P(A) \leqslant \sum P\left(A_{i}\right)
$$

which underlies all the bounds. Secondly, if an upper bound improving on (1) is available then by the same method tighter successive lower and upper bounds may be obtained. This technique is illustrated by parlaying an upper bound of Worsley (1982) into a lower bound. This lower bound is then calculated for a runs problem in Schwager (1984) in which the usual lower Bonferroni bounds fail to perform adequately.

\section{Methodology}

We begin with the equation

$$
P(A)=P\left(A_{1}\right)+P\left(A_{2} \overline{A_{1}}\right)+\cdots+P\left(A_{n} \overline{A_{1}} \overline{A_{2}} \cdots \overline{A_{n-1}}\right) .
$$

By expanding the general term and using (1) we get

$$
\begin{aligned}
P\left(A_{i} \overline{A_{1}} \cdots \overline{A_{i-1}}\right) & =P\left(A_{i}\right)-P\left(A_{i} A_{1} \cup A_{i} A_{2} \cup \cdots \cup A_{i} A_{i-1}\right) \\
& \geqslant P\left(A_{i}\right)-\left[P\left(A_{i} A_{1}\right)+\cdots+P\left(A_{i} A_{i-1}\right)\right]
\end{aligned}
$$


and then collecting terms we are led to

$$
P(A) \geqslant \sum P\left(A_{i}\right)-\sum_{j<i} P\left(A_{i} A_{j}\right)
$$

the second Bonferroni inequality. For the next one we again expand the general term as above but this time we use (3), which we have just proven, to derive the second line of (2). This gives

$$
P\left(A_{i} \overline{A_{1}} \cdots \overline{A_{i-1}}\right) \leqslant P\left(A_{i}\right)-\left[\sum_{j<i} P\left(A_{i} A_{j}\right)-\sum_{k<j<i} P\left(A_{i} A_{i} A_{k}\right)\right]
$$

which results in

$$
P(A) \leqslant \sum P\left(A_{i}\right)-\sum_{j<i} P\left(A_{i} A_{j}\right)+\sum_{k<j<i} P\left(A_{i} A_{j} A_{k}\right) .
$$

In this fashion each bound is obtained from the preceding one.

\section{An improved lower bound}

Worsley (1982) presents the following sharpening of (1). Represent the events $\left\{A_{i}\right\}$ as vertices $\left\{v_{i}\right\}$ of a graph $G$ where $v_{i}$ and $v_{j}$ are connected with an edge $e_{i}$ if and only if $A_{i} A_{j} \neq \emptyset$. Let $H$ be a subgraph of $G$. Then

$$
P(A) \leqslant \sum P\left(A_{i}\right)-\sum_{\left\{e_{i}, \in H\right\}} P\left(A_{i} A_{j}\right)
$$

if and only if $H$ is a tree. If $G$ is a tree and $H=G$ then there is equality in (4).

From (4) Worsley obtains bounds derived by Kounias (1968) and Kwerel (1975) as well as the interesting corollary

$$
P(A) \leqslant \sum_{i=1}^{n} P\left(A_{i}\right)-\sum_{i=1}^{n-1} P\left(A_{i} A_{i+1}\right) .
$$

We now present an improved lower bound based on (5).

\section{Theorem}

$$
P(A) \geqslant \sum_{i=1}^{n} P\left(A_{i}\right)-\sum_{m<i} P\left(A_{i} A_{m}\right)+\sum_{i=3}^{n} \sum_{m=1}^{i-2} P\left(A_{m} A_{m+1} A_{i}\right) .
$$

Proof. Consider what happens if (5) is used in place of (1) in the argument of Section 2. We get

$$
\begin{aligned}
P\left(A_{i} \overline{A_{1}} \overline{A_{2}} \ldots \overline{A_{i-1}}\right) & =P\left(A_{i}\right)-\left[P\left(A_{i} A_{1} \cup \ldots \cup A_{i} A_{i-1}\right)\right] \\
& \geqslant P\left(A_{i}\right)-\sum_{m=1}^{i-1} P\left(A_{i} A_{m}\right)+\sum_{m=1}^{i-2} P\left(A_{m} A_{m+1} A_{i}\right)
\end{aligned}
$$

which results in (6).

It is possible to obtain lower bounds based on (4) and on the upper bounds of Kounias and Kwerel but we have chosen to focus on (5) for simplicity of expression. 


\section{Numerical example}

Schwager (1984) considers a sequence $\left\{X_{1}, X_{2}, \ldots, X_{n}\right\}$ of independent and identically distributed Bernoulli random variables where $p=P\left[X_{1}=1\right]$ and $1-p=P\left[X_{1}=0\right]$. Given $k$ let $N=n-k+1$, $A_{i}=\left\{X_{i}=X_{i+1}=\cdots=X_{i+k-1}=1\right\}$ and $A=\cup_{i=1}^{N} A_{i}$, the event that the sequence contains a string of at least $k$ successive 1's. Schwager computes the first two upper bounds $S_{1}, S_{3}$, the first Worsley bound $S_{1}^{*}$ (the right-hand side of (5)), the first two lower bounds $S_{2}, S_{4}$ and compares them with the exact values of $P(A)$ for $p=0.5,0.6, k=10,15,20$ and $n=100,300,500,1000,1500$. He shows that $S_{1}^{*}$ approximates $P(A)$ very well in most of these cases and is always much better than $S_{1}$ or $S_{3}$. The bounds $S_{2}$ and $S_{4}$, however, are all poor and in many cases negative.

We have calculated the right-hand side of (6), denoted by $S_{2}^{*}$, and in all the above cases $S_{2}^{*}$ offers improvement over the lower bounds similar to what $S_{1}^{*}$ does for the upper bounds (as well as providing the

Table 1

\begin{tabular}{|c|c|c|c|c|}
\hline$n$ & $S_{2}$ & $S_{2}^{*}$ & Exact & $S_{1}^{*}$ \\
\hline \multicolumn{5}{|c|}{$p=0.5, k=10$} \\
\hline 100 & -0.00106 & 0.04338 & 0.04414 & 0.04492 \\
\hline 300 & -0.03530 & 0.12382 & 0.13351 & 0.14258 \\
\hline 500 & -0.10768 & 0.18519 & 0.21452 & 0.24023 \\
\hline 1000 & -0.45553 & 0.25517 & 0.38545 & 0.48437 \\
\hline 1500 & -1.04181 & 0.20593 & 0.51918 & 0.72852 \\
\hline \multicolumn{5}{|c|}{$p=0.5, k=15$} \\
\hline 100 & 0.00006 & 0.00133 & 0.00133 & 0.00133 \\
\hline 300 & 0.00003 & 0.00437 & 0.00437 & 0.00438 \\
\hline 500 & -0.00004 & 0.00738 & 0.00741 & 0.00743 \\
\hline 1000 & -0.00038 & 0.01484 & 0.01495 & 0.01506 \\
\hline 1500 & -0.00094 & 0.02219 & 0.02244 & 0.02269 \\
\hline \multicolumn{5}{|c|}{$p=0.5, k=20$} \\
\hline 100 & 0.000002 & 0.00004 & 0.00004 & 0.00004 \\
\hline 300 & 0.000002 & 0.00013 & 0.00013 & 0.00013 \\
\hline 500 & 0.000002 & 0.00023 & 0.00023 & 0.00023 \\
\hline 1000 & 0.000001 & 0.00047 & 0.00047 & 0.00047 \\
\hline 1500 & 0.000001 & 0.00071 & 0.00071 & 0.00071 \\
\hline \multicolumn{5}{|c|}{$p=0.6, k=10$} \\
\hline 100 & -0.36660 & 0.17634 & 0.20491 & 0.22372 \\
\hline 300 & -2.28017 & -0.13211 & 0.51606 & 0.70745 \\
\hline 500 & -5.65620 & -0.49709 & 0.70545 & 1.19118 \\
\hline 1000 & -20.49455 & -4.62941 & 0.91487 & 2.40051 \\
\hline 1500 & -44.47330 & -12.41790 & 0.97540 & 3.60983 \\
\hline \multicolumn{5}{|c|}{$p=0.6, k=15$} \\
\hline 100 & -0.01898 & 0.01624 & 0.01637 & 0.01646 \\
\hline 300 & -0.07347 & 0.05084 & 0.05278 & 0.05407 \\
\hline 500 & -0.13681 & 0.08190 & 0.08785 & 0.09169 \\
\hline 1000 & -0.33383 & 0.14408 & 0.16994 & 0.18572 \\
\hline 1500 & -0.58612 & 0.18415 & 0.24465 & 0.27976 \\
\hline \multicolumn{5}{|c|}{$p=0.6, k=20$} \\
\hline 100 & -0.00135 & 0.00120 & 0.00121 & 0.00121 \\
\hline 300 & -0.00504 & 0.00412 & 0.00412 & 0.00413 \\
\hline 500 & -0.00880 & 0.00700 & 0.00703 & 0.00706 \\
\hline 1000 & -0.01841 & 0.01412 & 0.01427 & 0.01437 \\
\hline 1500 & -0.02836 & 0.02111 & 0.02145 & 0.02168 \\
\hline
\end{tabular}


same degree of accuracy). The computations are presented in Table 1 which repeats the relevant parts of Table 1 of Schwager (1984) as well as the values of $S_{2}^{*}$.

$S_{2}^{*}$ is computed as follows. $S_{2}^{*}=S_{2}+\Delta_{2}$ where

$$
\Delta_{2}=\sum_{i=3}^{N} \sum_{m=1}^{i-2} P\left(A_{m} A_{m+1} A_{i}\right) .
$$

If $m+k<i$ then $P\left(A_{m} A_{m+1} A_{j}\right)=p^{2 k+1}$ while if $m+k \geqslant i$ then $P\left(A_{m} A_{m+1} A_{i}\right)=p^{k+i-m}$. Thus

$$
\begin{aligned}
\Delta & =\sum_{i=3}^{k+1} \sum_{m=1}^{i-2} P\left(A_{m} A_{m+1} A_{i}\right)+\sum_{i=k+2}^{N} \sum_{m=1}^{i-k-1} P\left(A_{m} A_{m+1} A_{i}\right)+\sum_{i=k+2}^{N} \sum_{m=i-k}^{i-2} P\left(A_{m} A_{m+1} A_{i}\right) \\
& =\sum_{i=3}^{k+1} \sum_{m=1}^{i-2} p^{i+k-m}+\sum_{i=k+2}^{N} \sum_{m=1}^{i-k-1} p^{2 k+1}+\sum_{i=k+2}^{N} \sum_{m=i-k}^{i-2} p^{k+i-m}=a+b+c
\end{aligned}
$$

where

$$
a=p^{k}\left[(k-1) p^{2}-\left(p^{3}-p^{k+2}\right) /(1-p)\right] /(1-p), \quad b=p^{2 k+1}(N-k-1)(N-k) / 2
$$

and

$$
c=\left(p^{k+2}-p^{2 k+1}\right)(N-k-1) /(1-p) .
$$

These complicated expressions fail to expose what $S_{2}^{*}$ really is. Observe that

$$
\begin{aligned}
\Delta_{2} & =\sum_{i=3}^{N} \sum_{m=1}^{i-2} P\left(A_{m} A_{m+1} A_{i}\right)=p \sum_{i=3}^{N} \sum_{m=1}^{i-1} P\left(A_{m+1} A_{i}\right) \\
& =p \sum_{i=2}^{N-1} \sum_{m=1}^{i-1} P\left(A_{m} A_{i}\right) \quad \text { (by stationarity) } \\
& \equiv p \sum_{i=2}^{N} \sum_{m=1}^{i-1} P\left(A_{m} A_{i}\right) \quad \text { (approximately) } \\
& =p\left(S_{1}-S_{2}\right) .
\end{aligned}
$$

Thus $S_{2}^{*}=S_{2}+p\left(S_{1}-S_{2}\right)=p S_{1}+(1-p) S_{2}$, a linear combination of $S_{1}$ and $S_{2}$.

It is possible to derive a second upper bound by the method of Section 3 namely

$$
P(A) \leqslant S_{3}^{*}=S_{3}-\Delta_{3}
$$

where

$$
\Delta_{3}=\sum_{r=4}^{N} \sum_{i=3}^{r-1} \sum_{m=1}^{i-2} P\left(A_{m} A_{m+1} A_{i} A_{r}\right) .
$$

As in the previous paragraph we can show that

$$
\Delta_{3} \equiv p\left(S_{3}-S_{2}\right)
$$

and $S_{3}^{*} \cong p S_{2}+(1-p) S_{3}$. Unfortunately this bound does not improve on $S_{1}^{*}$. 


\section{References}

Feller, W. (1968), An Introduction to Probability Theory and its Applications, Vol. I (John Wiley \& Sons, New York, 3rd ed.).

Kounias, E.G. (1968), Bounds for the probability of a union, Ann. Math. Statist. 39, 2154-2158.
Kwerel, S.M. (1975), Most stringent bounds on aggregated probabilities of partially specified dependent probability systems, J. Amer. Statist. Assoc. 70, 472-479.

Schwager, S.J. (1984), Bonferroni sometimes loses, Amer. Statist. 38, 192-197.

Worsley, K.J. (1982), An improved Bonferroni inequality and applications, Biometrika 69, 297-302. 